\title{
A Unit Cell Structure of Smooth Surfaces with Auxetic Response
}

\author{
Lin $\mathrm{YU}^{1}$, Hui-Feng TAN ${ }^{1,2}$, a, Teng $\mathrm{MA}^{1}$ and Zhen-Gong $\mathrm{ZHOU}^{1,2}$ \\ ${ }^{1}$ Centre for Composite Materials and Structures, Harbin Institute of Technology, \\ ${ }^{2}$ National Key Laboratory of Science and Technology on Advanced Composites in Special \\ Environments, Harbin Institute of Technology,
}

Harbin, China

Keywords: Cellular structure, Auxetic, Mechanical properties, Periodic boundary condition.

\begin{abstract}
A unit cell structure of smooth surfaces was designed in this paper. By combining the orientations of the convex of the surfaces, four different models were presented. The mechanical properties of the models were studied by numerical analysis. The techniques of the representative volume element and the periodic boundary conditions were applied in the analysis. Little differences in the mechanical properties between the models with opposite convex orientations are found. The simulation results showed that the auxetic responses of the unit cell are universal at variant the geometric variables and parent material Poisson's ratios. The design concept is a highly effective method to fabricate cellular structures with similar frameworks.
\end{abstract}

\section{Introduction}

Auxetic materials response in the opposite way as the ordinary materials. Under uniaxial loading, auxetic materials would contract if compressed and expand if stretched. Some natural materials will display auxetic responses [1-6]. Since the first artificial auxetic foam manufactured by Lakes [7], a variety of auxetic materials [8-11] have been investigated and fabricated by different methods.

Some 3D structures, such as Bucklicrystals and buckyball like shells [12, 13] who gain auxetic from elastic buckling, cannot act as basic framework due to the loss of the stiffness. In this paper, a unit cell build up by smooth surfaces is presented. The mechanical properties and the auxetic response are studied by numerical analysis. The techniques of periodic boundary conditions and the representative volume element are used in the investigation to reduce the complexity of the model and the computation time consumption. The design concept is such a versatile procedure that a diverse 3D configurations can be designed in a similar way.

\section{Structure of the Unit Cell}

The line-element-replacing procedure is a prevalent way to designing $2 \mathrm{D}$ auxetic structures $[8,10$, 14], and exploiting the analogous way a unit cell of smooth surfaces is designed. In this paper, a unit cell structure made up of six identical wavy surfaces is presented. Each surface has a constant thickness of $t$ and the formula of the surface is

$$
z=A \sin \left(\frac{2 \pi}{L} x\right) \sin \left(\frac{2 \pi}{L} y\right)
$$

where $A$ is the amplitude of the surface and $L$ is the dimension in $x$ and $y$ axis. Obviously, the surface is a smooth one by simple mathematical calculation. The unit cell of smooth surfaces (Figure 1a) is obtained by replacing the six surfaces of a cube of edge length $L$ with the surface aforementioned, which is called cubic instance. Neglecting the convex orientation, an equivalent instance, called treble cross instance, is shown in Figure 1b, which consist three surfaces intersecting each other mutual orthogonally through the centers of the surfaces. 

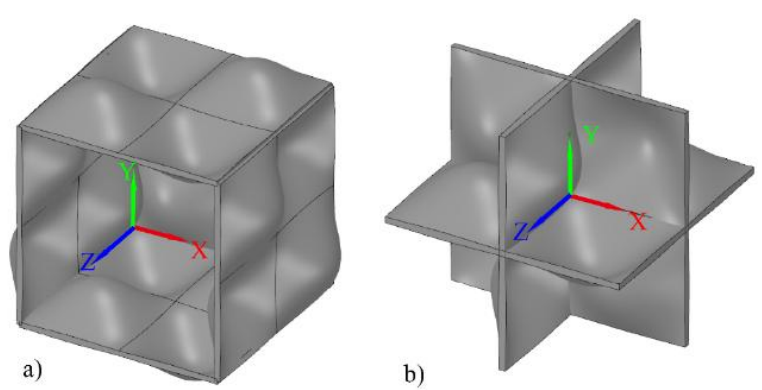

Fig. 1. Two equivalent instances of the unit cell. a) cubic instance; b) treble cross instance

As only half of each surface in cubic instance belongs to the unit and the other half consist the neighbor unit. So two instance have the identical relative density. The relative density of unit cell is the ratio of the unit density to the solid from which it is made, or equivalently the volume fraction of surfaces occupying the unit cell [15]. The volume fraction is more clearly in the treble cross instance, and the volume of one surfaces is

$$
V_{1}=A_{s u r} t
$$

where the $A_{\text {sur }}$ is the area of the surface and is given by

$$
\begin{aligned}
& A_{\text {sur }}=\int_{0}^{L} \int_{0}^{L}\left(1+z_{x}^{2}+z_{y}^{2}\right)^{1 / 2} d x d y \\
& =\int_{0}^{L} \int_{0}^{L}\left(1+\frac{4 \pi^{2} A^{2}}{L^{2}}\left(\sin ^{2} \frac{2 \pi x}{L} \cos ^{2} \frac{2 \pi y}{L}+\cos ^{2} \frac{2 \pi x}{L} \sin ^{2} \frac{2 \pi y}{L}\right)\right)^{1 / 2} d x d y
\end{aligned}
$$

Considering the intersection of the surfaces, the volume should be updated as

$$
V=3 V_{1}-3 L t^{2}=3\left(A_{\text {sur }}-L t\right) t
$$

So the relative density is

$$
\rho_{r}=\frac{V}{L^{3}}=3\left(\frac{A_{\text {sur }}}{L^{2}}-\frac{t}{L}\right) \frac{t}{L}
$$

So the geometry of the unit cell of smooth surface can be characterized by two dimensionless parameters: the area coefficient, $A_{\text {sur }} / L^{2}$, and the normalized thickness, $t / L$.

By comparison of two instances, we found that it is more convenient to apply the loads and boundary conditions on the instance a. And in the following simulations, the cubic instance is chosen to be the default instance in the numerical analysis and discussion.

We assume that the surfaces on the parallel surfaces of the cube have the same convex orientations for simplification. For the three surface lying on the coordinate plane, the notation " + " is assigned to the surface if the convex nearest to the origin $\mathrm{O}$ is pointing outside of the cube and the notation "-" is assigned if pointing inside.

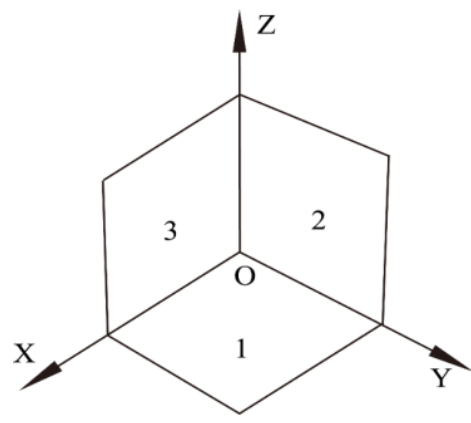

Fig. 2. The illustration of the cube position and the surface number. 
Placing the unit cell in the first octant and viewing in the angle from the point $(1,1,1)$ to origin $O$, all the combinations of the notations are listed in the Table 1 in the order of surface number 1,2, 3 (Figure 2). Four different models are obtained by changing and combining the notations.

Table 1. Four models and their configuration notations.

\begin{tabular}{|c|c|c|c|c|}
\hline Model & A & B & C & D \\
\hline 1 & + & + & + & - \\
\hline 2 & + & + & - & - \\
\hline 3 & + & - & - & - \\
\hline
\end{tabular}

\section{Method}

Mechanical behavior of the four models with different normalized thickness under uniaxial tension have been studied using the commercial finite element software Abaqus/Standard. Linear full integrated 4-node doubly curved shell elements (Abaqus element type S4) are generated in all model. In simulation, the parent material is modeled as linear elastic with Young's modulus $E_{\mathrm{m}}=$ $1.5 \mathrm{GPa}$ and Poisson's ratio $v_{\mathrm{m}}=0.35$. The normalized thickness variation from 0.005 to 0.1 was considered in the numerical simulations and the interval thickness fall into an approximately logarithmic distribution. The default amplitude is chosen as $3.0 \mathrm{~mm}$. And the default thickness is chosen as $1.0 \mathrm{~mm}$ with a corresponding normalized thickness of 0.05 .

In the analysis, uniaxial tension load is applied as a uniform displacement, $\delta y$ of $0.1 \mathrm{~mm}$ in $y$ direction causing a homogenized strain $\bar{\varepsilon}_{y y}$ of $0.5 \%$. To avoid rigid motion of model, vertex $O$ is fixed by setting the displacement in $z$ direction to zero additionally. Then the periodic boundary conditions are applied on parallel edges and corresponding vertices [16].

To characterize the mechanical properties of the unit cell, the effective stiffness is defined as

$$
k=\frac{f}{\delta_{y}}
$$

where $f$ is the reaction force in $y$ direction in simulation.

To quantify the lateral deformation of the unit cell, we calculate the Poisson's ratio over the unit cell. First the averaged displacements of the surface is obtained by averaging the nodal displacements of the edges. Then the average strain values for the unit cell are derived as

$$
\varepsilon_{x x}=\frac{\left\langle u_{x}\right\rangle^{\mathrm{R}}-\left\langle u_{x}\right\rangle^{\mathrm{L}}}{L}, \varepsilon_{y y}=\frac{\left\langle u_{x}\right\rangle^{\mathrm{F}}-\left\langle u_{x}\right\rangle^{\mathrm{B}}}{L}, \varepsilon_{z z}=\frac{\left\langle u_{x}\right\rangle^{\mathrm{U}}-\left\langle u_{x}\right\rangle^{\mathrm{D}}}{L}
$$

where R, L, F, B, U, D, denote right, left, front, back, up and down boundaries of unit cell.

So the negative ratio of $\varepsilon_{x x}, \varepsilon_{y y}$ and $\varepsilon_{z z}$ are calculated to characterized the macroscopic Poisson's ratio

$$
v_{y x}=-\frac{\varepsilon_{x x}}{\varepsilon_{y y}}, v_{y z}=-\frac{\varepsilon_{z z}}{\varepsilon_{y y}} .
$$

To keep the integrity of the assembly, each unit in the assembly undergo the same deformation mode and there is no separation or overlap between the neighboring unit cells. Compatibility of the stress and strain fields should be ensured under periodic boundary conditions, which can be expressed as displacement continuous condition and stress continuous condition [16].

The periodic boundary conditions of a surface, for example the rear surface, is demonstrated as follows. 


\section{Displacement Continuous Condition}

Displacement continuous condition implies that each unit has the same deformation and do not separate or overlap each other during the deformation.

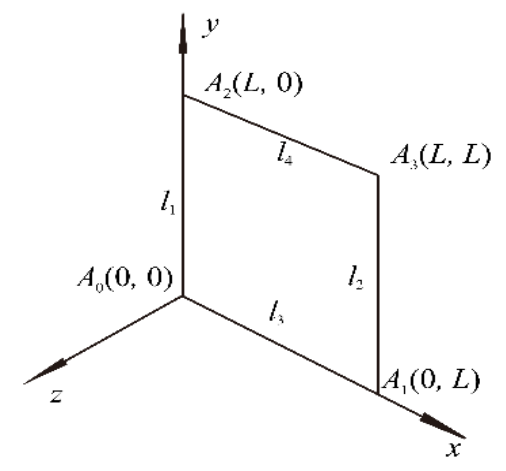

Fig. 3. The geometrical frame of the rear surface

For a rear surface as shown in Figure 3, the displacements on the opposite edges are

$$
\begin{aligned}
& \boldsymbol{u}(x, L)=\boldsymbol{u}(x, 0)+\bar{\varepsilon}_{y} L \\
& \boldsymbol{u}(L, y)=\boldsymbol{u}(0, y)+\bar{\varepsilon}_{x} L
\end{aligned}
$$

where $\boldsymbol{u}(x, y)$ means the displacement vector at location $(x, y)$ and $\bar{\varepsilon}_{i}$ means the average strain in $i$ direction, $i=x, y$.

For a pair of edges $\bar{\varepsilon}_{i}$ is constant, therefore the displacement continuous condition is obtained as follows:

$$
\begin{aligned}
& \boldsymbol{u}(x, L)-\boldsymbol{u}(x, 0)=c_{y} \\
& \boldsymbol{u}(L, y)-\boldsymbol{u}(0, y)=c_{x}
\end{aligned}
$$

where $c_{x}=\bar{\varepsilon}_{x} L, c_{y}=\bar{\varepsilon}_{y} L$. For the convenience in the simulation,

$$
\begin{aligned}
& c_{x}=\boldsymbol{u}_{A_{2}}-\boldsymbol{u}_{A_{0}} \\
& c_{y}=\boldsymbol{u}_{A_{1}}-\boldsymbol{u}_{A_{0}}
\end{aligned}
$$

are often utilized where $\boldsymbol{u}_{A_{i}}$ are the displacement of the corresponding vertices.

\section{Stress Continuous Condition}

For the constrained rear surface, variation of work by an arbitrary virtual boundary displacement $\left(d W_{1}\right)$ should be equal to the virtual work performed by the external loads on the retained boundary of the RVE $\left(d W_{2}\right)$. Virtual work $d W_{1}$ could be expressed by the Cauchy stress tensor $\sigma$ and the outward normal unit $\boldsymbol{n}$ on the boundary $\Gamma$ as

$$
d W_{1}=\int_{\Gamma}(\boldsymbol{\sigma} \cdot \boldsymbol{n}) \delta \boldsymbol{u} d \Gamma
$$

External force $\boldsymbol{f}_{A_{i}}$ acting on vertex $A_{i}$ and distributed external load $\boldsymbol{p}_{l_{i}}$ along the retained boundary $l_{i}$ are utilized to expressed virtual work $d W_{2}$ as

$$
d W_{2}=\int_{l_{1}} \boldsymbol{p}_{l_{1}} \delta \boldsymbol{u}(0, y) d l_{1}+\int_{l_{3}} \boldsymbol{p}_{l_{3}} \delta \boldsymbol{u}(x, 0) d l_{3}+\sum_{i=1}^{4} \boldsymbol{f}_{A_{i}} \delta \boldsymbol{u}_{A_{i}}
$$

Substituting Eq. (10) and Eq. (11) into Eq. (12) and comparing the corresponding coefficient of 
each virtual displacement along the boundary and vertex between the Eq. (12) and Eq. (13), external loads are obtained as:

$$
\begin{gathered}
\boldsymbol{f}_{A_{0}}=\int_{l_{1}}(\boldsymbol{\sigma} \cdot \boldsymbol{n}) d \Gamma+\int_{l_{3}}(\boldsymbol{\sigma} \cdot \boldsymbol{n}) d \Gamma \\
\boldsymbol{f}_{A_{1}}=-\int_{l_{1}}(\boldsymbol{\sigma} \cdot \boldsymbol{n}) d \Gamma \\
\boldsymbol{f}_{A_{2}}=0 \\
\boldsymbol{f}_{A_{3}}=-\int_{l_{3}}(\boldsymbol{\sigma} \cdot \boldsymbol{n}) d \Gamma \\
\boldsymbol{p}_{l_{1}}=\boldsymbol{\sigma} \cdot \boldsymbol{n}_{l_{1}}+\boldsymbol{\sigma} \cdot \boldsymbol{n}_{l_{2}} \\
\boldsymbol{p}_{l_{3}}=\boldsymbol{\sigma} \cdot \boldsymbol{n}_{l_{3}}+\boldsymbol{\sigma} \cdot \boldsymbol{n}_{l_{4}}
\end{gathered}
$$

On the opposite boundary of surface, stress vectors should be opposite to ensure the compatibility, so

$$
\begin{gathered}
\boldsymbol{\sigma} \cdot \boldsymbol{n}_{l_{1}}=-\boldsymbol{\sigma} \cdot \boldsymbol{n}_{l_{2}} \\
\boldsymbol{\sigma} \cdot \boldsymbol{n}_{l_{2}}=-\boldsymbol{\sigma} \cdot \boldsymbol{n}_{l_{4}} .
\end{gathered}
$$

From Eq. (18) and Eq. (19), we achieve that

$$
\begin{aligned}
& \boldsymbol{p}_{l_{1}}=0 \\
& \boldsymbol{p}_{l_{2}}=0
\end{aligned}
$$

Equation (21) denote that external distributed loads on the boundary vanish. All the formula above shows that external loads are equivalent to the concentrated loads applied on the vertices. So the displacements are only applied on the vertices $A_{0}, A_{1}$ and $A_{2}$ of the surface under periodic boundary conditions.

The periodic boundary conditions on the edges of the other 5 surfaces can be applied in a similar way as the edges on the rear surface.

\section{Result and Discussion}

Utilizing the numerical model mentioned above, the compression proprieties of the unit cell of four different models are obtained and discussed as follow. In consideration of the linear material model and linear analysis technique, the results are also efficacious for compression loading. The auxetic responses are found in all of the four models.

It is worthy to note that little differences between the model A and D, B and C are found for variant parental modulus, Poisson's ratio and normalized thickness by comparing the curves in the Figure 4, 5 and 6. This phenomenon indicate that the convex orientation has little influence on the mechanical properties if the parallel surfaces have the same orientations.

\section{The Effect of Parental Modulus}

Like other auxetic systems resulting from the micro structures [12], macroscopic Poisson's ratio $v_{y x}, v_{y z}$ are independent of the modulus of the bulk material [15]. That means that the auxetic is totally induced from the micro structures, i.e. the configurations of the curve surfaces in the cube are fundamental to the auxetic responses. 


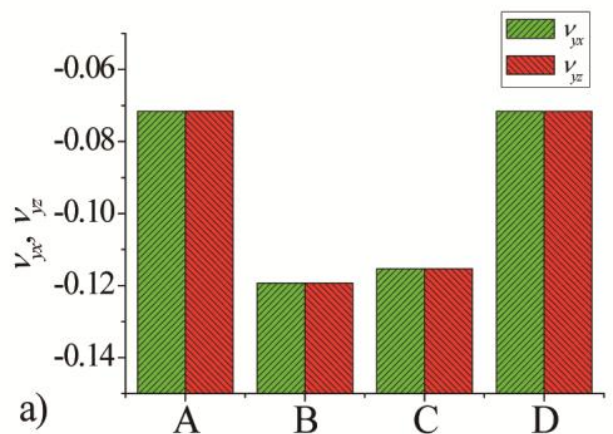

Fig. 4. a) Macroscopic Poisson's ratio of four models and b) the effective stiffness plot against the $E_{\mathrm{m}}$.

As we can see from the Figure 4a, the macroscopic Poisson's ratio $v_{y x}, v_{y z}$ of four models are equal, meaning that the lateral contractions in $x$ and $z$ direction are identical. So the two macroscopic Poisson's ratio $v_{y x}, v_{y z}$ can be discussed as one variable and $v_{y x}$ is used in the following discussion.

Figure $4 \mathrm{~b}$ shows that the evolutions of effective stiffness as the parental modulus are linearly. No difference of the effective stiffness between four models can be seen which implying that the convex configuration do not affect the axial reactions in the loading directions.

\section{The Effect of Parental Poisson's Ratio}

As the unit cell is made up of ordinary material with a positive Poisson's ratio, so the macroscopic Poisson's ratio deduced from the lateral contraction under loading would be attenuated by the nonauxetic response of the parental material. This effect can be observed in Figure 5a and all of the four models display auxetic responses for a thermodynamic stable range of parental Poisson's ratio, $(-1,0.5)$ [17]. As the $v_{m}$ approaches $-1(\mathrm{G} \rightarrow \infty)$, the unit cell exhibited a similar shear resisting response as the parental material.

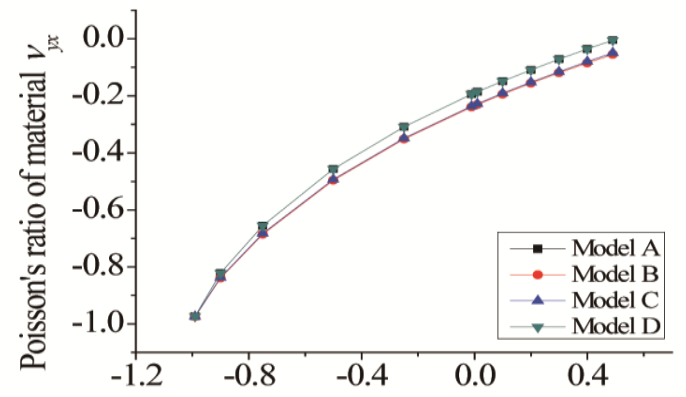

a)

Poisson's ratio of material $v_{m}$

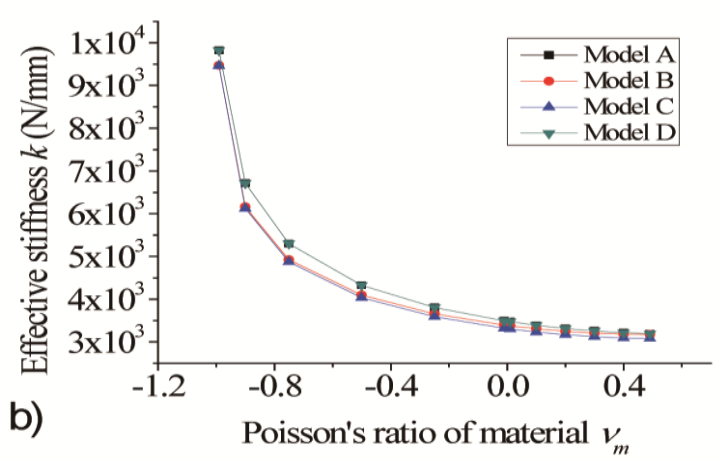

Fig. 5. a) Macroscopic Poisson's ratio plot against the $v_{\mathrm{m}}$ and b) the effective stiffness plot against the $v_{\mathrm{m}}$.

Figure 5b shows the evolution of the effective stiffness as a function of the parental Poisson's ratio. The response of all models is characterized by a sharp decline and a following a plateau. As the parental Poisson's ratio turn to positive, i.e. the bulk material act as ordinary material, the change of the effective stiffness is much smaller.

\section{The Effect of the Normalized Thickness}

The normalized thickness changes the crossing area of the unit cell changes linearly. So does the 
mechanical property of the unit cell. The dependence of the macroscopic Poisson's ratio on the normalized thickness of the surfaces can be seen from the Figure 6a. As the normalized thickness increases from $0.01 \mathrm{~mm}$ to $1.0 \mathrm{~mm}$, the absolute value of the macroscopic Poisson's ratio get smaller (less auxetic) from 0.23 to about 0.1 . It is rather remarkable that all the models with variant normalized thicknesses show auxetic responses. The bigger normalized thickness conduce a more evident discrepancy between the model A, D and model B, C. The effective stiffness shows a linear evolution as a function of the normalized thickness, which is consistent with the basic knowledge of mechanics of materials, shown as the following relational expression.

$$
k=\frac{f}{\delta_{y}} \propto A_{\text {crossing }} \propto \frac{t}{L}
$$

a)

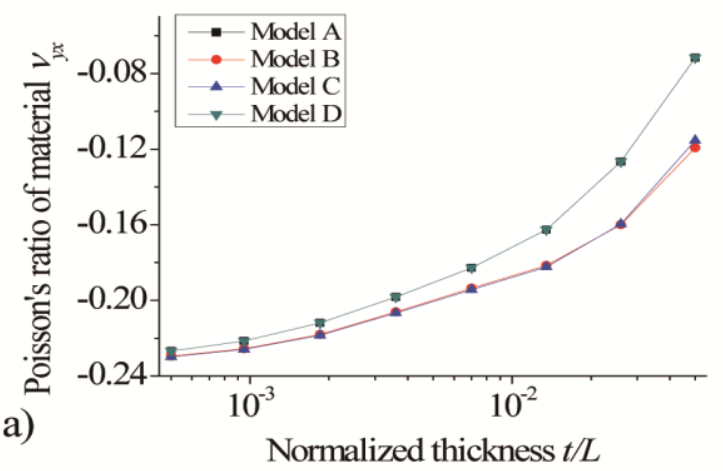

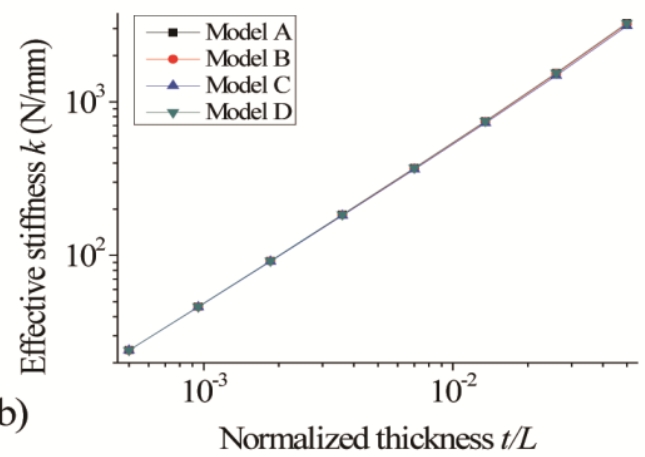

Fig. 6. a) Macroscopic Poisson's ratio plot against $t / L$ and b) the effective stiffness plot against the $t / L$

As discussed before, the default normalized thickness is chosen as 0.05 when the auxetic response of the unit cell is in the worst case. So unit cell with more auxetic response is easy to achieve by selecting the material more conscientiously.

\section{Conclusion}

The present work proposed a unit cell structure of smooth surfaces with auxetic response by replacing the surfaces of a cube. The geometry properties of the unit cell are given by analytical methods. Four different models are generated by combining the convex orientations of the surfaces. By imposing periodic boundary conditions on a selected RVE, the effects of mechanical properties on the convex orientations, parent material properties and the geometric parameter are studied by numerical analysis. The mechanical properties of the unit cells with same convex orientation on parallel surfaces is independent of the convex orientation. The phenomenon that all the four models show auxetic response indicates the high effectiveness of the design concept.

\section{Acknowledgement}

This work was supported by the National Natural Science Foundations of China (Grant No. 11272105) and the Fundamental Research Funds for the Central Universities (Grant No. HIT.MKSTISP. 201609).

\section{References}

1. R. Gatt, M. V. Wood, A. Gatt, F. Zarb, C. Formosa, K. M. Azzopardi, A. Casha, T. P. Agius, P. Schembri-Wismayer, L. Attard, N. Chockalingam, J. N. Grima, Acta Biomater 24,201(2015).

2. D. R. Veronda and R. A. Westmann, J Biomech 3, 111 (1970).

3. V. L. Coenen and K. L. Alderson, Phys Status Solidi (b) 248, 66 (2011). 
4. J. L. Williams and J. L. Lewis, J Biomech Eng-T ASME 104, 50 (1982).

5. A. Nur and G. Simmons, Earth Planet SC Lett 7, 183 (1969).

6. R. V. Goldstein and V. A. Gorodtsov and D. S. Lisovenko, Dokl Phys 57, 174 (2012).

7. R. Lakes, Science 1038, 235 (1987).

8. Q. Ma, H. Cheng, K. Jang, H. Luan, K. Hwang, J. A. Rogers, Y. Huang, and Y. Zhang, J Mech Phys Solids 90, 179 (2016).

9. D. Li and L. Dong and R. S. Lakes, Mater Lett 456, 164 (2016).

10. Y. Sun and N. Pugno, Materials 6, 699 (2013).

11. K. Jang, H. U. Chung, S. Xu, C. H. Lee, H. Luan, J. Jeong, H. Cheng, G. Kim, S. Y. Han, J. W. Lee, J. Kim, M. Cho, F. Miao, Y. Yang, H. N. Jung, M. Flavin, H. Liu, G. W. Kong, K. J. Yu, S. I. Rhee, J. Chung, B. Kim, J. W. Kwak, M. H. Yun, J. Y. Kim, Y. M. Song, U. Paik, Y. Zhang, Y. Huang, J. A. Rogers, Nat Commun 6, 6566 (2015).

12. S. Babaee, J. Shim, J. C. Weaver, E. R. Chen, N. Patel, and K. Bertoldi, Adv Mater 25, 5044 (2013).

13. J. Shim, C. Perdigou, E. R. Chen, K. Bertoldi, and P. M. Reis, P Natl Acad Sci USA, 109, 5978 (2012).

14. D. Mousanezhad, S. Babaee, H. Ebrahimi, R. Ghosh, A. S. Hamouda, K. Bertoldi, and A. Vaziri, Sci Rep-UK 5, 18306 (2015).

15. R. Biagi and H. Bart-Smith, Int J Solids Struct 44, 4690 (2007).

16. Z. Xia and Y. Zhang and F. Ellyin, Int J Solids Struct 40, 1907 (2003).

17. F. Javid, E. Smith-Roberge, M. C. Innes, A. Shanian, J. C. Weaver, and K. Bertoldi, Sci RepUK 5, 18373 (2015). 